\title{
The complete genome sequence of the methanogenic archaeon ISO4-H5 provides insights into the methylotrophic lifestyle of a ruminal representative of the Methanomassiliicoccales
}

Yang Li ${ }^{1,2}$, Sinead C. Leahy ${ }^{1 *}$, Jeyamalar Jeyanathan ${ }^{1}$, Gemma Henderson ${ }^{1}$, Faith Cox ${ }^{1}$ Eric Altermann ${ }^{1}$, William J. Kelly ${ }^{1}$, Suzanne C. Lambie ${ }^{1}$, Peter H. Janssen ${ }^{1}$, Jasna Rakonjac ${ }^{2}$ and Graeme T. Attwood ${ }^{1 *}$

\begin{abstract}
Methane emissions from agriculture represent around $9 \%$ of global anthropogenic greenhouse emissions. The single largest source of this methane is animal enteric fermentation, predominantly from ruminant livestock where it is produced mainly in their fermentative forestomach (or reticulo-rumen) by a group of archaea known as methanogens. In order to reduce methane emissions from ruminants, it is necessary to understand the role of methanogenic archaea in the rumen, and to identify their distinguishing characteristics that can be used to develop methane mitigation technologies. To gain insights into the role of methylotrophic methanogens in the rumen environment, the genome of a methanogenic archaeon has been sequenced. This isolate, strain ISO4-H5, was isolated from the ovine rumen and belongs to the order Methanomassiliicoccales. Genomic analysis suggests ISO4-H5 is an obligate hydrogen-dependent methylotrophic methanogen, able to use methanol and methylamines as substrates for methanogenesis. Like other organisms within this order, ISO4-H5 does not possess genes required for the first six steps of hydrogenotrophic methanogenesis. Comparison between the genomes of different members of the order Methanomassiliicoccales revealed strong conservation in energy metabolism, particularly in genes of the methylotrophic methanogenesis pathway, as well as in the biosynthesis and use of pyrrolysine. Unlike members of Methanomassiliicoccales from human sources, ISO4-H5 does not contain the genes required for production of coenzyme $M$, and so likely requires external coenzyme $M$ to survive.
\end{abstract}

Keywords: Methanogen, Methane, Ruminant, Methanomassiliicoccales, Pyrrolysine

Abbreviations: bp, Base pair; Cdc, Cell Division Control Protein; COG, Cluster of Orthologous Groups; CoM, Coenzyme M; CRISPR, Clustered Regularly Interspaced Short Palindromic Repeat; Fpo, $F_{420}$ Methanophenazine Oxidoreductase; kb, Kilobase; Mb, Megabase; MCL, Maximum Composite Likelihood; Mrt, Methyl Coenzyme M Reductase II

\footnotetext{
* Correspondence: sinead.leahy@agresearch.co.nz; graeme.attwood@ agresearch.co.nz

${ }^{1}$ AgResearch Limited, Grasslands Research Centre, Palmerston North, New

Zealand

Full list of author information is available at the end of the article
} 


\section{Introduction}

Ruminant animals have evolved a digestive system in which microbes in their rumen break down plant fiber and provide fermentation end-products and other nutrients for growth and development of the animal [1]. The rumen is densely populated with bacteria, archaea, ciliate protozoa, anaerobic fungi and viruses which participate in complex interactions to bring about the digestion of forage material. The archaeal community is made up almost exclusively of methanogens, which use simple energy sources such as hydrogen, formate and methyl compounds and produce methane. Rumen methanogens play an important role in preventing the accumulation of hydrogen derived from microbial fermentation of plant polysaccharides. This allows reduced cofactors, generated during microbial fermentation, to be reoxidised so that the main fiber-degrading function of the rumen can continue. The methane formed from this process is belched from the animal to the atmosphere, where it contributes a global warming potential (over 100 years, $\mathrm{GWP}_{100}$ ) of around $34 \times$ that of carbon dioxide [2, 3]. The production of methane represents a loss of energy from the ruminant, and depending on the diet, this loss can represent 3.8 to $12.8 \%$ of energy contained in the diet [4-6].

Methanogens are classified into three broad categories based on the compounds they use for methanogenesis: hydrogenotrophic, methylotrophic and acetoclastic [7]. In the rumen, methane is formed mainly via the hydrogenotrophic and methylotrophic pathways. Members of the new order of methanogenic archaea, Methanomassiliicoccales, are hydrogen-dependent methylotrophic methanogens and have been detected in various habitats, including landfills, rice fields, marine thermal vents, fresh water, and in the digestive tracts of termites, millipedes, chickens, ruminants and humans [8-18]. The Methanomassiliicoccales are considered to be an important group in the rumen environment and were originally referred to as Rumen Cluster $C$ methanogens [19, 20]. Their abundance in the rumen is highly variable, according to $16 S$ ribosomal RNA gene surveys [21-23], but on average, they are the second most abundant order of rumen methanogens and constitute around $16 \%$ of the rumen archaeal community based on clone library analyses [24], and $13 \%$ of rumen archaeal community based on pyrosequencing [25]. Representatives of these organisms have only recently been isolated in culture, and genomic information on members of the Methanomassiliicoccales are available only for isolates from human, bovine [26-29] and termite sources (NCBI Reference Sequence: NC_020892.1). This study reports the complete genome sequence of an ovine rumen member of Methanomassiliicoccales, designated methanogenic archaeon ISO4-H5.

\section{Organism information}

\section{Classification and features}

A methane-forming enrichment culture was originally obtained from a 9-year-old Romney wether sheep in New Zealand grazing a ryegrass-clover pasture diet [30]. The enrichment culture contained the methanogenic archaeon, ISO4-H5, and a Gram-negative bacterium, subsequently identified as being closely related to Succinivibrio dextrinosolvens and designated as strain $\mathrm{H} 5$. The methanogenic archaeon ISO4-H5 grows slowly and requires 3 to 4 days to generate detectable methane in the culture headspace. The optical density of cultures after maximal methane formation is very low and ISO4-H5 cells cannot be visualized via fluorescence microscopy at $420 \mathrm{~nm}$ due to the apparent lack of the fluorescent 8hydroxy-5-deazaflavin cofactor, known as $F_{420}$ [30]. The organism has only a thin bi-layer cell membrane, and no S-layer or cell wall was observed in electron micrographs of thin sections of ISO4-H5 cells (Fig. 1). The $16 S$ ribosomal RNA gene of ISO4-H5 is $96 \%$ identical to "Candidatus Methanomethylophilus alvus" Mx1201 enriched from human feces [27], and $95 \%$ identical to Thermoplasmatales archaeon BRNA1 enriched from bovine rumen (Fig. 2). All three are members of the order Methanomassiliicoccales, but potentially each represent different species [31]. The general features of methanogenic archaeon ISO4-H5 are shown in Table 1 and Additional file 1: Table S1.

\section{Genome sequencing information}

\section{Genome project history}

To gain insight into the role of methylotrophic methanogens in the rumen environment, the genome of the methanogenic archaeon isolate ISO4-H5 was sequenced. Methanogenic archaeon isolate ISO4-H5 represents the

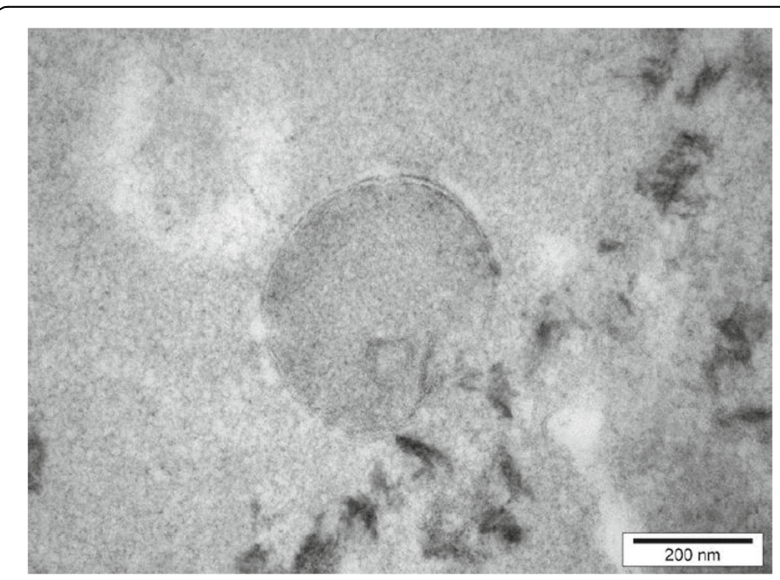

Fig. 1 Transmission electron micrograph of negatively stained thin section of the methanogenic archaeon ISO4-H5. The sample was prepared as previously described [60]. Images were captured using a Philips CM10 Transmission Electron Microscope, using an Olympus SIS Morada camera and SIS iTEM software (Germany) 


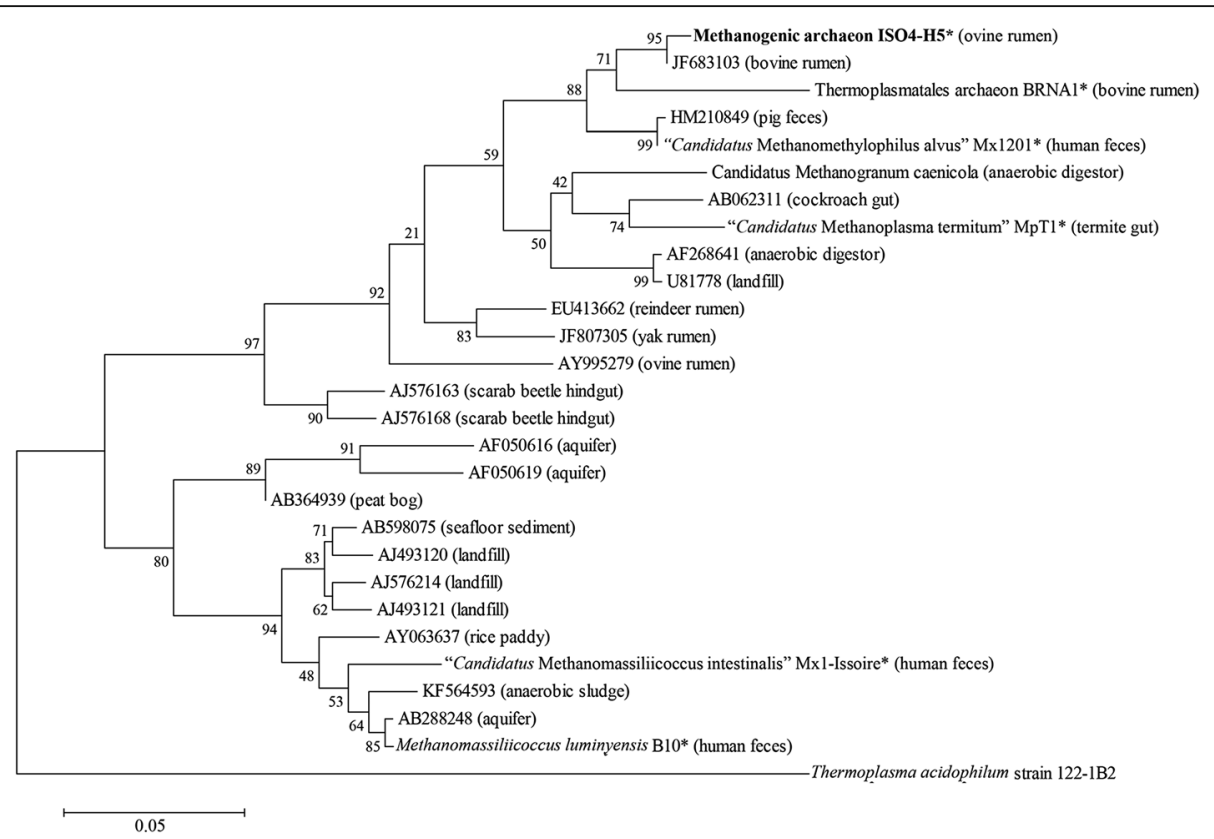

Fig. 2 Phylogenetic analysis of Methanomassiliicoccales 165 rRNA gene sequences. Phylogenetic tree showing the relationships of methanogenic archaeon ISO4-H5 (shown in bold print) relative to other type and non-type strains within the order Methanomassiliicoccales. The phylogeny was inferred from 165 rRNA gene nucleotide sequences (1474 bp internal region) aligned using the Maximum Likelihood method based on the Kimura 2-parameter model [61]. Evolutionary analyses were conducted in MEGA6 [62]. The bootstrap consensus tree from 1000 replicates [63] was used to infer the evolutionary history of the taxa analysed. Bar: 0.05 substitutions per nucleotide position. The GenBank accession numbers of environmental sequences are displayed with the source habitat given in brackets. Strains whose genomes have been sequenced are marked with an asterisk. The initial tree for the heuristic search was obtained by applying the Neighbor-Joining method to a matrix of pairwise distances estimated using the Maximum Composite Likelihood (MCL) approach. All positions containing gaps or missing data were eliminated, giving a total of 455 positions in the final dataset. The 165 rRNA gene sequence from Thermoplasma acidophilum 122-1B2 was used as an outgroup

first genome sequence of a member of the order Methanomassiliicoccales isolated from the ovine rumen. A summary of the genome project information is shown in Table 2.

\section{Growth conditions and genomic DNA preparation}

The initial enrichment cultures were obtained by inoculation of sheep rumen contents into BY medium [32] upplemented with (final concentrations), SL10 trace elements solution $(1 \mathrm{~mL} / \mathrm{L})$ [33], selenite/tungstate solution $(1 \mathrm{~mL} / \mathrm{L})$ [33], sodium acetate $(20 \mathrm{mM})$, sodium formate $(60 \mathrm{mM})$, methanol $(20 \mathrm{mM})$, vitamin 10 solution (0.1 ml per $10 \mathrm{~mL}$ culture tube) [32], and coenzyme $\mathrm{M}$ $(\mathrm{CoM})(10 \mu \mathrm{M})$ [34]. The last two additives were added to the sterilized medium from filter-sterilized stock solutions. Hydrogen $\left(\mathrm{H}_{2}\right)$ was supplied as the energy source by pumping the culture vessels to $180 \mathrm{kPa}$ over pressure with an 80:20 mixture of $\mathrm{H}_{2}$ : carbon dioxide $\left(\mathrm{CO}_{2}\right)$. ISO4-H5 was enriched in tubes receiving sheep rumen contents diluted by a factor of 16,384,000 [30]. Several approaches were used to reduce the bacteria in the enrichment culture, including a 10-fold dilution, the addition of antibiotics (combinations of streptomycin, ampicillin, bacitracin at $10 \mu \mathrm{g} / \mathrm{mL}$ each, and vancomycin at $86.7 \mu \mathrm{g} / \mathrm{mL}$ ), heat treatment of the enrichment culture at $50{ }^{\circ} \mathrm{C}$ for 10 to $30 \mathrm{~min}$, and application of lysozyme $(2.5 \mathrm{mg} / \mathrm{mL})$. These approaches produced a limited diversity enrichment culture containing ISO4-H5 and $S$. dextrinosolvens $\mathrm{H} 5$, which was verified by phase contrast epifluorescence microscopy and bacterial and archaeal $16 S$ rRNA gene sequencing. Genomic DNA was extracted from cells harvested from a freshly grown $(7 \mathrm{~d}$ incubation time) $2 \mathrm{~L}$ enrichment culture using a modified version of a liquid $\mathrm{N}_{2}$ freezing and grinding method [35], in which treatment with $2.5 \mathrm{mg}$ lysozyme $/ \mathrm{mL}$ and $0.8 \mathrm{mg}$ proteinase $\mathrm{K} / \mathrm{mL}$ replaced the $1 \% \mathrm{w} / \mathrm{v}$ sodium dodecyl sulfate step, before a Genomic-tip 500/G (Qiagen, Germany) was used, following the manufacturer's instructions, in place of the phenol/chloroform extraction steps.

\section{Genome sequencing and assembly}

The DNA extracted from the ISO4-H5 enrichment culture was sequenced via pyrosequencing of a $3 \mathrm{~kb}$ mate 
Table 1 Classification and general features of the methanogenic archaeon ISO4-H5

\begin{tabular}{|c|c|c|c|}
\hline MIGS ID & Property & Term & Evidence code ${ }^{a}$ \\
\hline & Current classification & Domain: Archaea & TAS [64] \\
\hline & & Phylum: Euryarchaeota & TAS [65] \\
\hline & & Class: Thermoplasmata & TAS [66] \\
\hline & & Order: Methanomassiliicoccales & TAS $[66,67]$ \\
\hline & & Family: & \\
\hline & & Genus: & \\
\hline & & Species: & \\
\hline & & Strain: ISO4-H5 & TAS [30] \\
\hline & Gram stain & Not applicable & \\
\hline & Cell shape & Coccoid $0.3 \mu \mathrm{m} \sim 0.6 \mu \mathrm{m}$ diameter & \\
\hline & Motility & Non-motile & \\
\hline & Sporulation & Not spore-forming & IDA \\
\hline & Temperature range & Not reported & \\
\hline & Optimum temperature & $38 / 39^{\circ} \mathrm{C}$ & TAS [30] \\
\hline & $\mathrm{pH}$ range & Not reported & \\
\hline & Optimum pH & Not reported & \\
\hline & Carbon source & Not reported & \\
\hline & Energy source & $\mathrm{H}_{2}+$ methanol, mono-, di-, or trimethylamine & IDA \\
\hline & Terminal electron receptor & Methyl-substrates & IDA \\
\hline MIGS-6 & Habitat & Ovine rumen & TAS [30] \\
\hline MIGS-6.3 & Salinity & Not reported & \\
\hline MIGS-22 & Oxygen & Strict anaerobe & IDA \\
\hline MIGS-15 & Biotic relationship & Symbiont of ruminants & TAS [30] \\
\hline MIGS-14 & Pathogenicity & Not known as a pathogen & NAS \\
\hline MIGS-4 & Geographic location & Palmerston North, New Zealand & IDA \\
\hline MIGS-5 & Sample collection time & Autumn, 2008 & IDA \\
\hline MIGS-4.1 & Latitude & Latitude: -40.35 (40²1'00"S) & IDA \\
\hline MIGS-4.2 & Longitude & Longitude: +175.61 (175³6'36"E) & IDA \\
\hline MIGS-4.4 & Altitude & $30 \mathrm{~m}$ & IDA \\
\hline
\end{tabular}

${ }^{a}$ Evidence codes - TAS Traceable Author Statement (i.e., a direct report exists in the literature), IDA Inferred from Direct Assay, NAS Non-traceable Author Statement (i.e., not directly observed for the living, isolated sample, but based on a generally accepted property for the species, or anecdotal evidence). These evidence codes are from the Gene Ontology project [68]

paired-end sequence library using the 454 GS FLX platform with Titanium chemistry (Macrogen, Korea). Pyrosequencing reads provided $43.8 \times$ coverage of the combined ISO4-H5 and Succinivibrio dextrinosolvens H5 genomes, and were assembled using the Newbler assembler version 2.7 (Roche 454 Life Sciences, USA). The Newbler assembly resulted in 176 Succinivibrio dextrinosolvens $\mathrm{H} 5$ contigs across 28 scaffolds and 47 ISO4-H5 contigs in a single scaffold. The assignment of scaffolds to genomes was based on $\mathrm{G}+\mathrm{C}$ content analysis and identification of the methanogenesis marker gene, methyl coenzyme $M$ reductase $(m r t A)$. Sequence gap closure was managed using the Staden package [36] and gaps were closed using standard PCR techniques with Sanger sequencing. A total of 163 additional sequencing reactions were used to close gaps and to improve the quality of the genome sequence, ensuring correct assembly and to resolve base conflicts.

\section{Genome annotation}

Genome annotation was carried out as previously described [34, 37] and the ISO4-H5 genome sequence was prepared for NCBI submission using Sequin [38]. The guanosine residue of the start codon of the Cdc6-1 replication initiation protein gene (AR505_0001) was 
Table 2 ISO4-H5 genome project information

\begin{tabular}{lll}
\hline MIGS ID & Property & Term \\
\hline MIGS-31 & Finishing quality & High-quality, closed genome \\
MIGS-28 & Libraries used & 4543 kb mate paired-end library \\
MIGS-29 & Sequencing platforms & 454 GS-FLX Titanium chemistry \\
MIGS-31.2 & Fold coverage & $43.8 \times(454)$ \\
MIGS-30 & Assemblers & Newbler \\
MIGS-32 & Gene calling method & GLIMMER2.02 + BLASTX [69] \\
& Locus Tag & AR505 \\
& Genbank ID & CP014214 \\
& Genbank Date of Release & 12-February-2016 \\
& GOLD ID & Gp0125684 \\
& BIOPROJECT & PRJNA292473 \\
& BIOSAMPLE & SAMN03976563 \\
MIGS 13 & Source Material Identifier & Methanogenic archaeon ISO4-H5 \\
& Project relevance & Ruminant methane emissions \\
\hline
\end{tabular}

chosen as the first base for the ISO4-H5 genome. The nucleotide sequence of the ISO4-H5 chromosome has been deposited in Genbank under accession number CP014214.

\section{Genome properties}

The genome of ISO4-H5 consists of a single, $1,937,882 \mathrm{bp}$, circular chromosome with a $\mathrm{G}+\mathrm{C}$ content of $54 \%$. A total of 1,817 protein-coding genes were predicted, representing $90.2 \%$ of the total genome sequence. A Cluster of Orthologous Groups category was assigned to 1,434 of the protein-coding genes, and the properties of the genome are summarized in Tables 3 and 4 .

ISO4-H5 is predicted to contain two Cdc6 genes. Cdc6.1 (AR505_0001) is adjacent to two origin recognition box (ORB) motifs downstream [39], while Cdc6.2 (AR505_1205) is located $661 \mathrm{~kb}$ away from the Cdc6.1 gene and is not associated with any ORB motif. Therefore, Cdc6.1 is predicted to be the origin of replication for ISO4-H5 (Fig. 3). The presence of multiple origins of replications is a feature also observed in the genome sequences of other members of Methanomassiliicoccales, including BRNA1 (TALC00001, TALC00716, $645 \mathrm{~kb}$ apart), Mx1201 (MMALV_00010, MMALV_10400, $637 \mathrm{~kb}$ apart), Mx1-Issoire (H729_00005, H729_08750, $90 \mathrm{~kb}$ apart), and B10 (WP_019178385, WP_019178317). The ISO4-H5 genome contains genes predicted to be integrases (AR505_0313, 0669, 0931, 1543, 1570, 1640, 1697), as well as several Clustered Regularly Interspaced Short Palindromic Repeat (CRISPR) genes (AR505_1089 1095) associated with a CRISPR region containing 35 repeats (bases $1,153,894$ to $1,155,995$ ). There is evidence of
Table 3 ISO4-H5 genome nucleotide content and gene count

\begin{tabular}{llc}
\hline Attribute & Value & \% of total \\
\hline Genome size (bp) & $1,937,882$ & 100.00 \\
DNA coding (bp) & $1,747,977$ & 90.20 \\
G + C content (bp) & $1,046,533$ & 54.0 \\
DNA scaffolds & 1 & 100.00 \\
Total genes & 1,874 & 100.00 \\
Protein-coding genes & 1,817 & 96.95 \\
RNA genes & 54 & 2.29 \\
Pseudo genes & 3 & 0.16 \\
Genes in internal clusters & NA & \\
Genes with function prediction & 1113 & 59.39 \\
Genes assigned to COGs & 1,434 & 76.52 \\
Genes with Pfam domains & 396 & 21.13 \\
Genes with signal peptides & 157 & 8.38 \\
Genes with transmembrane helices & 352 & 18.78 \\
CRISPR repeats & 1 &
\end{tabular}

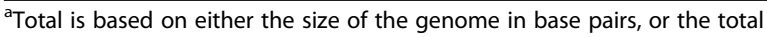
number of protein coding genes in the annotated genome

a mobile element in the ISO4-H5 genome (AR505_0313AR505_0358) which excised and segregated from the chromosome over several passages between the sequencing of the genome and subsequent analyses of the annotated locus. The $32 \mathrm{~kb}$ mobile element harbors 37 hypothetical protein genes, three adhesin-like protein genes, three DNA-cytosine methyltransferase genes, one phage integrase gene, one DNA mismatch endonuclease gene and one Membrane Occupation and Recognition Nexus (MORN) repeat-containing protein [40]. No plasmids were identified in the ISO4-H5 genome. The genome contains a predicted toxin/antitoxin module (AR505_0857, 0858) and a death-oncuring family protein (AR505_1566), although the latter lacks an identifiable gene encoding a partner toxin $[41,42]$.

\section{Insights from the genome}

The genomes of several members of Methanomassiliicoccales are publically available, including M. luminyensis B10 isolated from a human source, "Candidatus Methanomethylophilus alvus" Mx1201 and "Candidatus Methanomassiliicoccus intestinalis" Mx1-Issoire enriched from human sources, "Candidatus Methanoplasma termitum" MpT1 enriched from termite gut, and Thermoplasmatales archaeon BRNA1 enriched from the bovine rumen. These genomes were compared with ISO4-H5 (Table 5). ISO4-H5 is very similar in genome size to the other members of Methanomassiliicoccales, with $M$. 
Table 4 ISO4-H5 genes assigned to COG functional categories

\begin{tabular}{|c|c|c|c|}
\hline Code & value & $\%$ of total $^{a}$ & Description \\
\hline J & 144 & 7.89 & Translation \\
\hline A & 1 & 0.05 & RNA processing and modification \\
\hline K & 67 & 3.67 & Transcription \\
\hline L & 123 & 6.74 & Replication, recombination and repair \\
\hline B & 1 & 0.05 & Chromatin structure and dynamics \\
\hline D & 9 & 0.49 & Cell cycle control, mitosis and meiosis \\
\hline Y & 0 & 0.00 & Nuclear structure \\
\hline V & 17 & 0.93 & Defense mechanisms \\
\hline $\mathrm{T}$ & 20 & 1.10 & Signal transduction mechanisms \\
\hline M & 27 & 1.48 & Cell wall/membrane biogenesis \\
\hline N & 2 & 0.11 & Cell motility \\
\hline Z & 0 & 0.00 & Cytoskeleton \\
\hline W & 0 & 0.00 & Extracellular structures \\
\hline U & 13 & 0.71 & Intracellular trafficking and secretion \\
\hline O & 53 & 2.90 & $\begin{array}{l}\text { Posttranslational modification, protein } \\
\text { turnover, chaperones }\end{array}$ \\
\hline C & 87 & 4.77 & Energy production and conversion \\
\hline G & 40 & 2.19 & Carbohydrate transport and metabolism \\
\hline$E$ & 99 & 5.42 & Amino acid transport and metabolism \\
\hline $\mathrm{F}$ & 46 & 2.52 & Nucleotide transport and metabolism \\
\hline $\mathrm{H}$ & 105 & 5.75 & Coenzyme transport and metabolism \\
\hline । & 18 & 0.99 & Lipid transport and metabolism \\
\hline$P$ & 90 & 4.93 & Inorganic ion transport and metabolism \\
\hline Q & 14 & 0.77 & $\begin{array}{l}\text { Secondary metabolites biosynthesis, transport } \\
\text { and catabolism }\end{array}$ \\
\hline $\mathrm{R}$ & 242 & 13.26 & General function prediction only \\
\hline S & 126 & 6.90 & Function unknown \\
\hline - & 481 & 26.35 & Not in COGs \\
\hline
\end{tabular}

The total is based on the total number of protein coding genes in the annotated genome

luminyensis B10 being the exception, with a genome $35 \%$ larger than ISO4-H5. The genomic G + C content of the Methanomassiliicoccales range from 49 to $60 \%$, with "Candidatus Methanomassiliicoccus intestinalis" Mx1-Issoire being different to the rest with a genomic $\mathrm{G}+\mathrm{C}$ content of $41 \%$. The organization of genes within the ISO4-H5 genome shows best synteny with "Candidatus Methanomethylophilus alvus" Mx1201 and Thermoplasmatales archaeon BRNA1 (Fig. 4), its two closest genome-sequenced relatives.

\section{Methanogenesis}

Members of the order Methanomassiliicoccales rely solely on hydrogen-dependent methylotrophic methanogenesis to produce energy. However, they use only part of the pathway reported for other methylotrophic methanogens (Fig. 5), such as members of the genera Methanosarcina and Methanosphaera [43, 44]. Methanosarcina spp. disproportionate methanol by electron bifurcation, oxidizing one mole to produce $\mathrm{CO}_{2}$ while generating reducing potential to reduce three further moles to methane. The methanogenesis pathway in ISO4-H5 lacks the genes encoding the enzymes required to oxidize methanol to $\mathrm{CO}_{2}$, and is predicted to only reduce methylated compounds directly to methane. Functionally, this is similar to Methanosphaera stadtmanae MCB-3, which encodes all the genes for the enzymes needed to oxidize methanol to $\mathrm{CO}_{2}$ but does not use this pathway due to the lack of genes encoding synthesis of molybdopterin, a cofactor required for formation of an active formylmethanofuran dehydrogenase [44]. ISO4$\mathrm{H} 5$ is predicted to use a heterodisulfide reductase (HdrABC) and a methyl-viologen hydrogenase (MvhADG) to recycle $\mathrm{CoM}$, using reducing equivalents generated from the hydrogenase. However, unlike M. stadtmanae, the $\mathrm{Hdr}$ and Mvh complexes in ISO4-H5 are not predicted to be coupled to an energy-converting-hydrogenase complex [45], but rather are coupled to a $\mathrm{F}_{420}$-dehydrogenase Fpo-like complex to generate the membrane potential necessary for energy formation via ATP synthase $[46,47]$. The energy converting-hydrogenase complex identified in M. luminyensis B10 and "Candidatus Methanomassiliicoccus intestinalis" Mx1-Issoire could possibly have an anaplerotic role [48]. Based on the lack of the corresponding genes, the ISO4-H5 Fpo-like complex lacks the FpoF and FpoO subunits, which in other methanogens contain the iron-sulfur centers likely responsible for interacting with coenzyme $F_{420}$ and methanophenazine, respectively [49]. This is expected, as ISO4-H5 cells do not fluoresce when illuminated at $420 \mathrm{~nm}$, suggesting that coenzyme $F_{420}$ is not present in this organism. Furthermore, the genome does not contain genes for cytochrome biosynthesis, which suggests that methanophenazine is also absent. A hypothetical protein (AR505_1626) in the Fpo operon, between fpoK (AR505_1625) and fpoJ (AR505_1627) genes, is predicted to be a transmembrane protein and shares 49.5, 54.4 and $45.9 \%$ amino acid identity to MMALV_02020 of Mx1201, TALC_00216 of BRNA1 and Mpt1_c12590 of MpT1 respectively. In addition, this gene is also located in an operon whose organization is similar to those encoding BRNA1, Mx1201, and MpT1, and is possibly a subunit of the Fpo-like complex.

ISO4-H5 is predicted to have essentially the same methane formation pathway as "Candidatus Methanoplasma termitum" [29] and likely pumps only one ion across the cell membrane for every two methanes formed, to generate a membrane gradient. This is in 


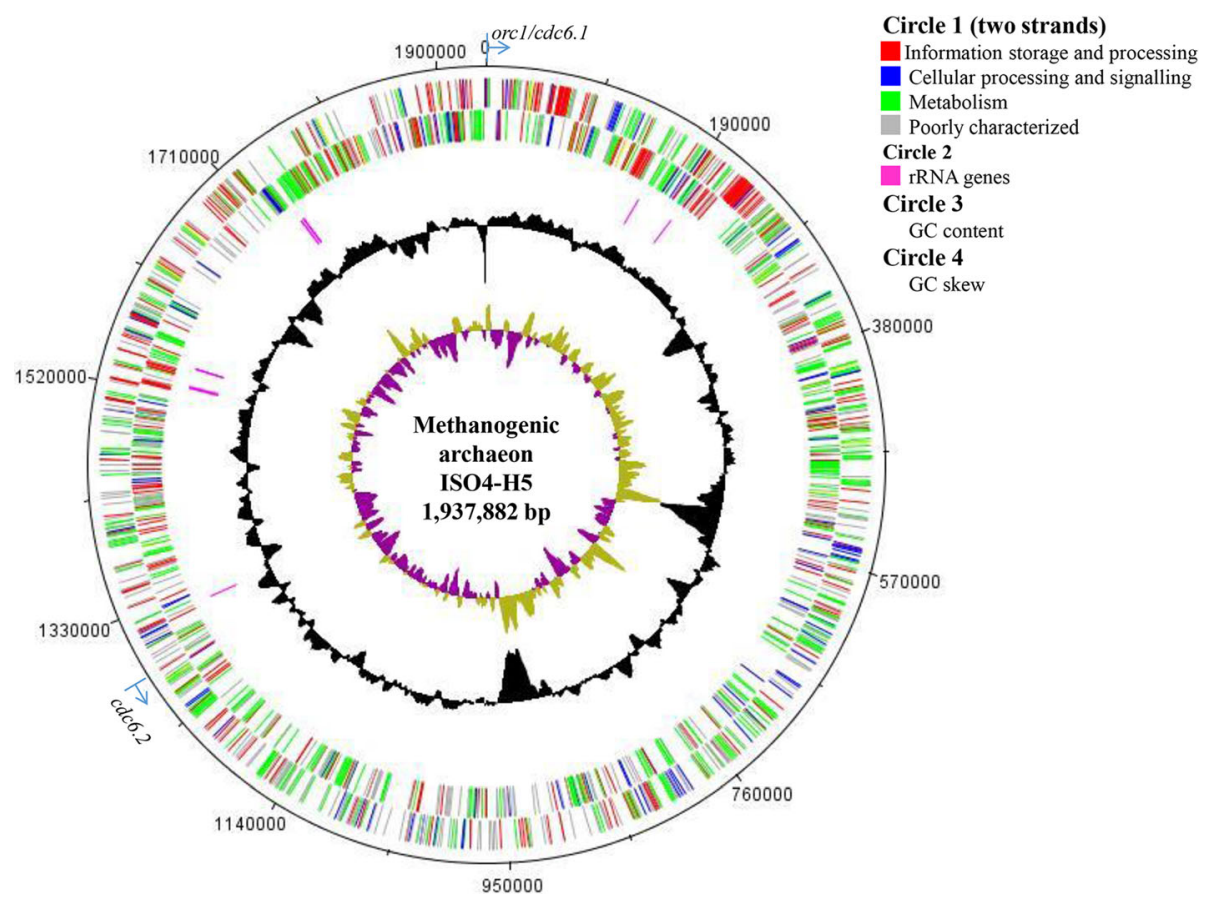

Fig. 3 Circular representation of ISO4-H5 genome. Circles are referred to as 1 (outermost) to 4 (innermost). Circle 1: predicted ORFs on the positive $(+)$ and negative $(-)$ strands, respectively. ORFs are colored based on the Clusters of Orthologous (COG) categories. Circle 2: location of the rRNA genes. Circle 3: $\% G+C$ content. Circle 4: $G C$ bias $[(G-C) /(G+C)]$, khaki indicates values $>1$, purple $<1$. This image was generated with DNA plotter [70]

contrast to M. stadtmanae, which has the same general metabolic stoichiometry but pumps two ions per methane formed [45]. Since ATP synthesis in all of these methanogens is via a membrane-bound ATP synthase, ISO4-H5 is predicted to have a have a much lower ATP (and growth) yield than Methanosphaera spp. which is consistent with the very low culture densities observed when the isolate is grown in the laboratory. However, it can be expected to have a lower threshold for hydrogen, using the same rationale proposed by Lang et al. (2015) for "Candidatus Methanoplasma termitum". This therefore differentiates it ecologically from Methanosphaera, and suggests that Methanosphaera spp. and members of
Methanomassiliiococcales, both of which occur in the rumen [24, 25], occupy different niches.

Interestingly, the cysteate synthase, cysteate aminotransferase $(\operatorname{ser} C)$ and sulfopyruvate decarboxylase (comDE) genes required for the synthesis of CoM [50] are absent from the ISO4-H5 genome. This suggests that ISO4-H5 cannot synthesize CoM, and requires an external supply of CoM to survive within the rumen, similar to Methanobrevibacter ruminantium M1 [34] and MpT1 [29]. This explains the requirement for CoM supplementation in the initial enrichments of ISO4-H5 [30]. ISO4-H5 also possesses only a subset of methanogenesis marker genes: 1-8, 11, 13, 15-17 (AR505_1391, 0786, 1390, 1417, 1388, 1389,

Table 5 Genomes of members of Methanomassiliicoccales from rumen and human sources

\begin{tabular}{|c|c|c|c|c|c|c|c|}
\hline Species & Status & Isolation source & Genome size (Mb) & Accession \# & CDS & $\% \mathrm{GC}$ & Reference \\
\hline Methanogenic archaeon ISO4-H5 & Complete & Ovine rumen & 1.94 & CP014214 & 1,823 & 54 & This report \\
\hline $\begin{array}{l}\text { Candidatus Methanomassiliicoccus } \\
\text { intestinalis Mx1-Issoire }\end{array}$ & Complete & Human feces & 1.93 & СР005934 & 1,876 & 41 & [26] \\
\hline $\begin{array}{l}\text { Candidatus Methanomethylophilus } \\
\text { alvus } \mathrm{M} \times 1201\end{array}$ & Complete & Human feces & 1.67 & СР004049 & 1,700 & 56 & [27] \\
\hline Methanomassiliicoccus luminyensis B10 & Draft & Human feces & 2.62 & $\begin{array}{l}\text { CAJE01000001 - } \\
\text { CAJE-1000026 }\end{array}$ & 2,669 & 60 & [28] \\
\hline Candidatus Methanoplasma termitum MpT1 & Complete & Termite gut & 1.49 & CP010070 & 1,415 & 49 & [29] \\
\hline Thermoplasmatales archaeon BRNA1 & Complete & Bovine rumen & 1.46 & CP002916 & 1,577 & 58 & Unpublished \\
\hline
\end{tabular}



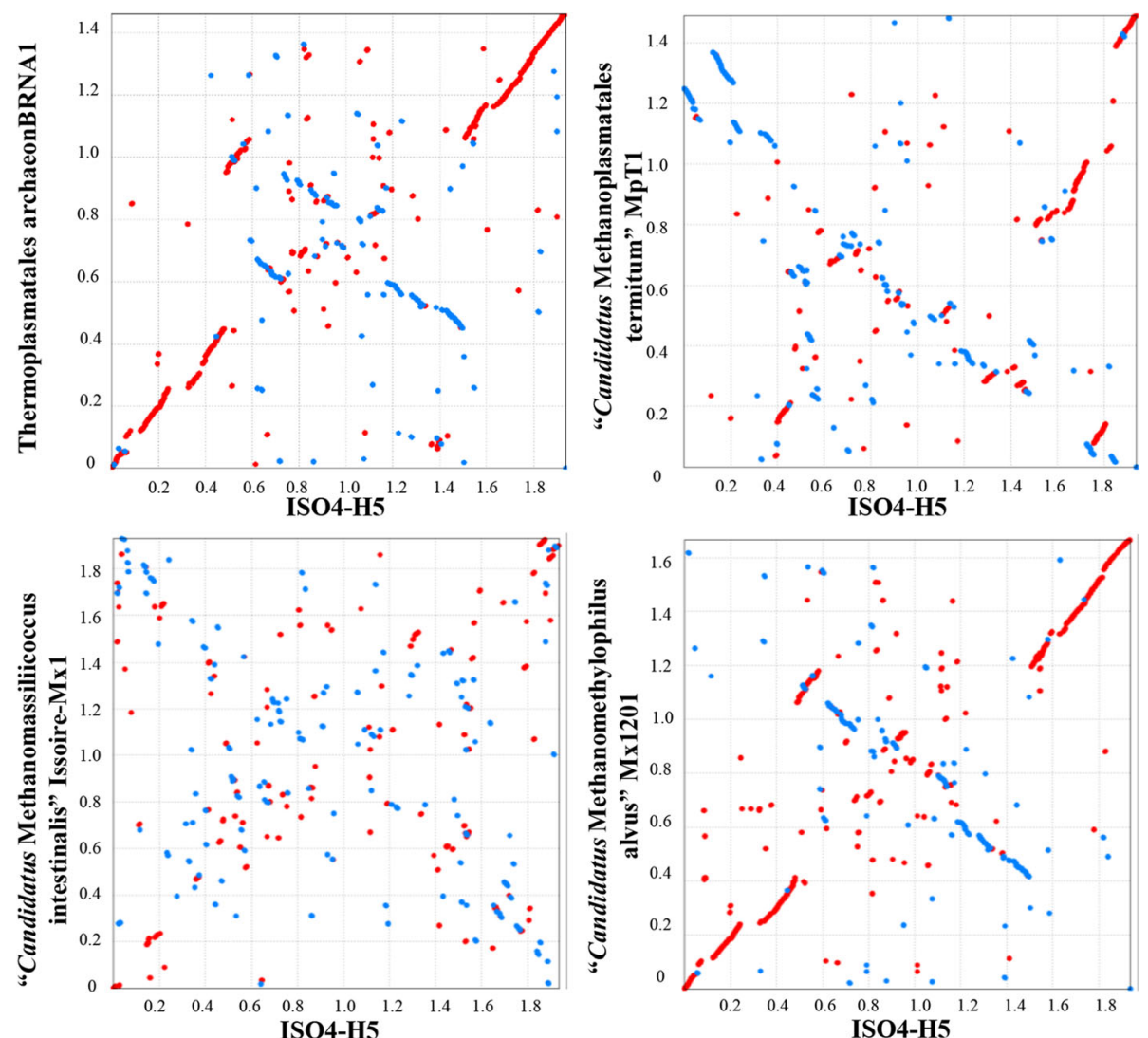

Fig. 4 Gene synteny plots for genomes of members of the order Methanomassiliicoccales. PROmer alignments of the ISO4-H5 genome against completed genomes from members of Methanomassiliicocaccales are shown. The alignments were plotted using MUMmer [71] with forward matches shown in red and reverse matches in blue. The units displayed on both axes are in million base pairs

$1385,1203,1637,0362,1387,0724$, and 1386 respectively). This suggests that the remaining methanogenesis marker genes (mmp 9, 10, 12 and 14) are not required for the truncated methyl-reducing pathway used by ISO4-H5.

\section{Pyrrolysine biosynthesis}

ISO4-H5 possesses a complete operon predicted to encode the genes required for the biosynthesis of pyrrolysine and for aminoacylation of a transfer RNA (tRNA) to pyrrolysine (Fig. 6) [51, 52], enabling read-through of the amber stop codon, UAG. Pyrrolysine is produced from two molecules of lysine by the gene products PylBCD. Methylornithine synthase (PylB) converts L-lysine to (3R)3 -methyl-D-ornithine, which in turn is ligated with a second molecule of L-lysine to produce $(2 R, 3 R)$-3-methylornithyl- $N^{6}$ lysine, catalysed by $(2 R, 3 R)-3$-methylornithyl$N^{6}$-lysine synthase (PylC); pyrrolysine synthase (PylD) converts $(2 R, 3 R)$-3-methylornithyl- $N^{6}$-lysine to pyrrolysine [53]. Pyrrolysine-tRNA ligase (PylS) catalyses the aminocylation of tRNA (CUA) which itself is encoded by pylT [54]. The operon organization is conserved across the Methanomassiliicoccales (Fig. 6), suggesting pyrrolysine use is important for members of this order. The in-frame amber codon occurs in 46 ISO4-H5 genes, including the genes encoding methylamine use; trimethylamine:corrinoid methyltransferase, mttB (AR505_0772); methanol corrinoid protein, $m t a C$ (AR505_0952); monomethylamine methyltransferase, mtmB (AR505_1327, 1328); and dimethylamine:corrinoid methyltransferase, $m t b B$ (AR505_1332). The amber codon is also found in the mmp 8 gene, a predicted nitrogenase gene (AR505_1289), an adenylate kinase gene (AR505_1784) involved in purine biosynthesis, a bifunctional phosphoglucose/phosphomannose isomerase gene (AR505_0560) involved in the last step of gluconeogenesis, two geranylgeranyl reductase genes (AR505_1433, AR505_1618) that are likely involved in cell membrane lipid biosynthesis, and the CRISPRassociated endonuclease Cas3 gene (AR505_1089) that is involved in acquired immunity against foreign DNA. Additionally, 17 genes encoding hypothetical proteins, one adhesin-like protein gene, and 10 insertion sequence elements have amber codons. Similar findings have been reported in the genomes of members of Methanomassiliicoccales of human origin and it has been suggested that pyrrolysine synthesis is a particular 


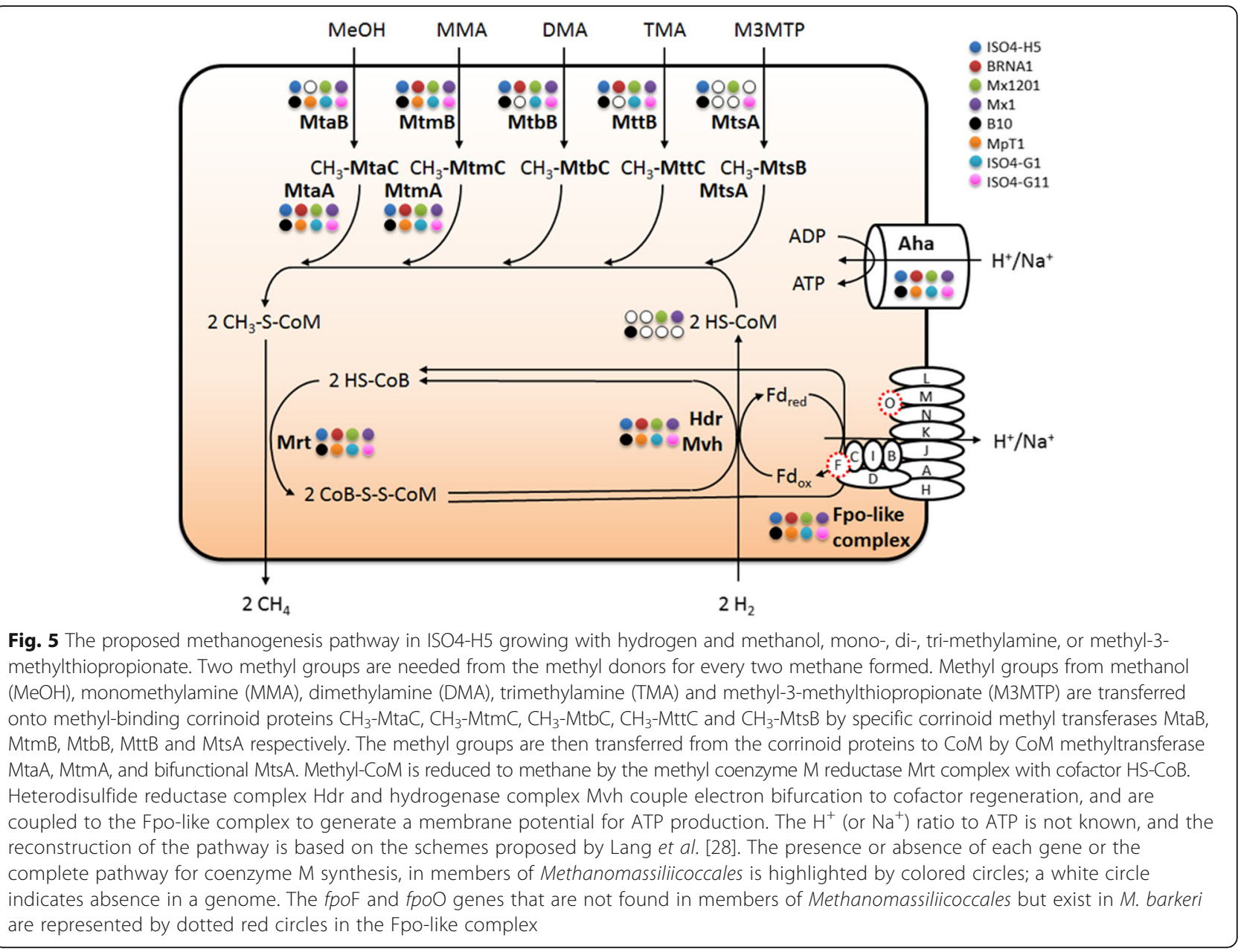

feature of this order and an important marker in the evolution of methanogenic archaea [55].

\section{Conclusions}

ISO4-H5 has a genome size of approximately $1.9 \mathrm{Mb}$, and a genomic $\mathrm{G}+\mathrm{C}$ content of $54 \%$, similar to the genomes of Mx1201, B10 and BRNA1. ISO4-H5 encodes the key genes and pathways required for hydrogendependent methylotrophic methanogenesis by reduction of methyl substrates, without the ability to oxidize methyl substrates to carbon dioxide. The wide range of methyl substrates predicted to be used by ISO4-H5 suggests it is more metabolically versatile than other methylotrophic methanogens within the rumen.

Members of Methanomassiliicoccales co-exist in the rumen with Methanosphaera spp. [24, 25, 56] and share similar substrate requirements. Methanomassiliicoccales are probably able to outcompete Methanosphaera in the rumen at low substrate concentrations, due to the lower thresholds conferred by the low ATP gain, but are probably disadvantaged when substrate concentrations are high and the low ATP yield limits their ability to proliferate. The variability of fermentation rates in the rumen associated with periods of feeding or fasting is therefore expected to give both groups of methylotrophic methanogens opportunities to grow.

ISO4-H5 appears to be reliant on the Hdr, Mvh and Fpo-like complexes for electron bifurcation, membrane potential generation and energy conservation, which is identical to what has been described in other members of Methanomassiliicoccales. However, ISO4-H5 is incapable of producing CoM, which suggests that ISO4$\mathrm{H} 5$ has adapted to the rumen environment, where CoM produced by other methanogens would be able to supplement ISO4-H5. ISO4-H5 also lacks the genes encoding cofactor $\mathrm{F}_{420}$ synthesis, rendering it nonfluorescent under illumination at $420 \mathrm{~nm}$. This trait has also been reported amongst other members of Methanomassiliicoccales, and is likely one of the key characteristics of this particular order of methanogens. However, a culture of B10 has been reported to fluoresce [57-59] and this may be consistent with B10 belonging to the deepest branching group within Methanomassiliicoccales [31]. 


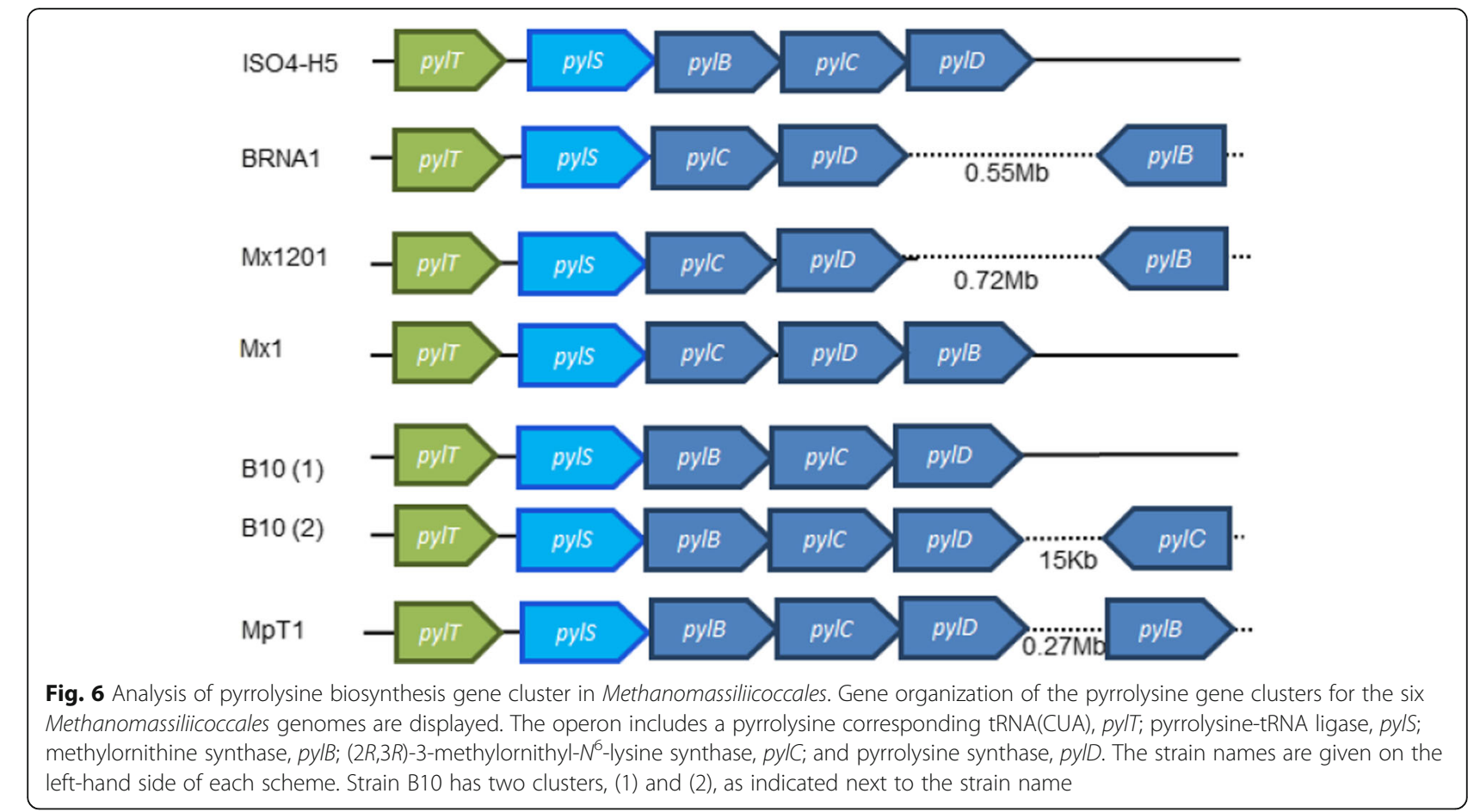

The use of pyrrolysine in proteins carrying out various cellular functions suggests it is important for ISO4-H5. While pyrrolysine is important in methylamine utilisation by all members of Methanomassiliicoccales sequenced thus far, pyrrolysine also appears to play a role in methanol use by ISO4-H5, as the methanol:methyltransferase corrinoid protein, $\mathrm{MtaC} 1$, is predicted to contain a pyrrolysine in its full length protein. The use of pyrrolysine and the Fpo-like complex by ISO4-H5 adds further weight to the hypothesis that the order Methanomassiliicoccales is evolutionary closer to the order Methanosarcinales, supporting findings from a previous phylogenetic study [24]. By analyzing the genome of ISO4-H5, our knowledge of the order Methanomassiliicoccales has been expanded, and together with the genomes of other members of the Methanomassiliicoccales, will be an important resource for the development of methane abatement technologies in ruminants.

\section{Additional file}

Additional file 1: Table S1. Associated MIGS record. (DOC 72 kb)

\section{Acknowledgements}

This work was funded by the New Zealand Agricultural Greenhouse Gas Research Centre (NZAGRC). We thank the Pastoral Greenhouse Gas Research Consortium (PGgRc) for providing access to the original enrichment cultures containing ISO4-H5. We thank Doug Hopcroft and Jordon Taylor (Manawatu Microscopy and Imaging Centre, Massey University) for technical assistance.
We thank Dong Li and Graham Naylor (AgResearch) for providing specialized technical advice.

\section{Authors' contributions}

SLe and GA initiated and supervised the study. JJ cultured the original enrichment containing ISO4-H5. YL, FC conducted the microbial culturing, SLa extracted genomic DNA, YL performed electron microscopy, assembled the genome, closed sequence gaps, annotated the genome and drafted the manuscript. SLe, GA, WK, GH, EA, PJ, JR discussed, analysed the data and revised the manuscript. All authors have read and approved the final manuscript.

\section{Competing interests}

The authors declare that they have no competing interests.

\section{Author details}

${ }^{1}$ AgResearch Limited, Grasslands Research Centre, Palmerston North, New Zealand. ${ }^{2}$ Institute of Fundamental Sciences, Massey University, Palmerston North, New Zealand.

Received: 16 February 2016 Accepted: 22 August 2016

Published online: 06 September 2016

\section{References}

1. Hungate RE. The rumen and its microbes. Michigan: Academic; 1966.

2. Boucher O. Comparison of physically- and economically-based $\mathrm{CO}_{2-}$ equivalences for methane. Earth Syst Dynam. 2012. doi:10.5194/esd-3-49-2012.

3. Joos F, Roth R, Fuglestvedt JS, Peters GP, Enting IG, von Bloh W, et al. Carbon dioxide and climate impulse response functions for the computation of greenhouse gas metrics: a multi-model analysis. Atmos Chem Phys. 2013. doi:10.5194/acp-13-2793-2013.

4. Johnson K, Huyler M, Westberg H, Lamb B, Zimmerman P. Measurement of methane emissions from ruminant livestock using a sulfur hexafluoride tracer technique. Environ Sci Technol. 1994. doi:10.1021/es00051a025.

5. Johnson KA, Johnson DE. Methane emissions from cattle. J Anim Sci. 1995;73:8.

6. Bell MJ, Eckard RJ. Reducing enteric methane losses from ruminant livestock-its measurement, prediction and the influence of diet. In: Khalid J, editor. Livestock Production. InTech; 2012. doi:10.5772/50394. 
7. Liu Y, Whitman WB. Metabolic, phylogenetic, and ecological diversity of the methanogenic archaea. Ann N Y Acad Sci. 2008. doi:10.1196/annals. 1419.019.

8. Egert M, Wagner B, Lemke T, Brune A, Friedrich MW. Microbial community structure in midgut and hindgut of the humus-feeding larva of Pachnoda ephippiata (Coleoptera: Scarabaeidae). Appl Environ Microbiol. 2003. doi:10. 1128/AEM.69.11.6659-6668.2003.

9. Großkopf R, Stubner S, Liesack W. Novel euryarchaeotal lineages detected on rice roots and in the anoxic bulk soil of flooded rice microcosms. Appl Environ Microbiol. 1998;64:12.

10. Horz HP, Seyfarth I, Conrads G. McrA and 165 rRNA gene analysis suggests a novel lineage of Archaea phylogenetically affiliated with Thermoplasmatales in human subgingival plaque. Anaerobe. 2012. doi:10.1016/j.anaerobe.2012.04.006.

11. Huang LN, Chen YQ, Zhou H, Luo S, Lan CY, Qu LH. Characterization of methanogenic Archaea in the leachate of a closed municipal solid waste landfill. FEMS Microbiol Ecol. 2003. doi:10.1016/S0168-6496(03)00218-6.

12. Iverson V, Morris RM, Frazar CD, Berthiaume CT, Morales RL, Armbrust EV. Untangling genomes from metagenomes: revealing an uncultured class of marine Euryarchaeota. Science. 2012. doi:10.1126/science.1212665.

13. Shinzato N, Matsumoto T, Yamaoka I, Oshima T, Yamagishi A. Phylogenetic diversity of symbiotic methanogens living in the hindgut of the lower termite Reticulitermes speratus analyzed by PCR and in situ hybridization. Appl Environ Microbiol. 1999;65:2.

14. Takai K, Horikoshi K. Genetic diversity of archaea in deep-sea hydrothermal vent environments. Genetics. 1999;152:4.

15. Dridi B, Henry M, Richet H, Raoult D, Drancourt M. Age-related prevalence of Methanomassiliicoccus luminyensis in the human gut microbiome. APMIS. 2012. doi:10.1111/j.1600-0463.2012.02899.x.

16. Padmanabha J, Liu J, Kurekci C, Denman SE, McSweeney CS. A methylotrophic methanogen isolate from the Thermoplasmatales affiliated RCC clade may provide insight into the role of this group in the rumen. In: Proceedings of the 5th Greenhouse Gases and Animal Agriculture Conference, Dublin. 2013.

17. Paul K, Nonoh JO, Mikulski L, Brune A. "Methanoplasmatales," Thermoplasmatalesrelated archaea in termite guts and other environments, are the seventh order of methanogens. Appl Environ Microbiol. 2012. doi:10.1128/AEM.02193-12.

18. Leahy SC, Kelly WJ, Ronimus RS, Wedlock N, Altermann E, Attwood GT. Genome sequencing of rumen bacteria and archaea and its application to methane mitigation strategies. Animal. 2013. doi:10.1017/ S1751731113000700.

19. Bapteste É, Brochier C, Boucher Y. Higher-level classification of the Archaea: Evolution of methanogenesis and methanogens. Archaea. 2005;1:5.

20. Tajima K, Nagamine T, Matsui H, Nakamura M, Aminov RI. Phylogenetic analysis of archaeal $16 \mathrm{~S}$ rRNA libraries from the rumen suggests the existence of a novel group of archaea not associated with known methanogens. FEMS Microbiol Lett. 2001. doi:10.1016/s0378-1097(01)00201-4.

21. Wright AD, Toovey AF, Pimm CL. Molecular identification of methanogenic archaea from sheep in Queensland, Australia reveal more uncultured novel archaea. Anaerobe. 2006. doi:10.1016/j.anaerobe.2006.02.002.

22. Zhou M, Hernandez-Sanabria E, Le LG. Assessment of the microbial ecology of ruminal methanogens in cattle with different feed efficiencies. Appl Environ Microbiol. 2009. doi:10.1128/aem.02815-08.

23. Huang XD, Tan HY, Long R, Liang JB, Wright AD. Comparison of methanogen diversity of yak (Bos grunniens) and cattle (Bos taurus) from the Qinghai-Tibetan plateau, China. BMC Microbiol. 2012. doi:10.1186/1471-2180-12-237.

24. Janssen $\mathrm{PH}$, Kirs M. Structure of the archaeal community of the rumen. Appl Environ Microbiol. 2008. doi:10.1128/aem.02812-07.

25. Henderson G, Cox F, Ganesh S, Jonker A, Young W, Janssen PH. Rumen microbial community composition varies with diet and host, but a core microbiome is found across a wide geographical range. Sci Rep. 2015. doi:10.1038/srep14567.

26. Borrel G, Harris HM, Parisot N, Gaci N, Tottey W, Mihajlovski A, et al. Genome sequence of "Candidatus Methanomassiliicoccus intestinalis" Issoire-Mx1, a third Thermoplasmatales-related methanogenic archaeon from human feces. Genome Announc. 2013. doi:10.1128/genomeA 00453-13

27. Borrel G, Harris HM, Tottey W, Mihajlovski A, Parisot N, Peyretaillade E, et al Genome sequence of "Candidatus Methanomethylophilus alvus" Mx1201, a methanogenic archaeon from the human gut belonging to a seventh order of methanogens. J Bacteriol. 2012. doi:10.1128/JB.01867-12.
28. Gorlas A, Robert C, Gimenez G, Drancourt M, Raoult D. Complete genome sequence of Methanomassiliicoccus luminyensis, the largest genome of a human-associated Archaea species. J Bacteriol. 2012. doi:10.1128/JB.00956-12.

29. Lang K, Schuldes J, Klingl A, Poehlein A, Daniel R, Brune A. New mode of energy metabolism in the seventh order of methanogens as revealed by comparative genome analysis of "Candidatus Methanoplasma termitum". Appl Environ Microbiol. 2015. doi:10.1128/AEM.03389-14.

30. Jeyanathan J. Investigation of rumen methanogens in New Zealand livestock [Thesis]. Palmerston North: Massey University; 2010.

31. Seedorf H, Kittelmann S, Henderson G, Janssen PH. RIM-DB: a taxonomic framework for community structure analysis of methanogenic archaea from the rumen and other intestinal environments. PeerJ. 2014. doi:10.7717/peerj.494.

32. Joblin KN, Naylor GE, Williams AG. Effect of Methanobrevibacter smithii on xylanolytic activity of anaerobic ruminal fungi. Appl Environ Microbiol. 1990;56:8.

33. Tschech A, Pfennig N. Growth yield increase linked to caffeate reduction in Acetobacterium woodii. Arch Microbiol. 1984. doi:10.1007/bf00414460.

34. Leahy SC, Kelly WJ, Altermann E, Ronimus RS, Yeoman CJ, Pacheco DM, et al. The genome sequence of the rumen methanogen Methanobrevibacter ruminantium reveals new possibilities for controlling ruminant methane emissions. PloS One. 2010. doi:10.1371/journal.pone.0008926.

35. Jarrell KF, Faguy D, Hebert AM, Kalmokoff ML. A general method of isolating high molecular weight DNA from methanogenic archaea (archaebacteria). Can J Microbiol. 1992;38:1.

36. Staden R, Beal KF, Bonfield JK. The Staden package, 1998. Methods Mol Biol. 2000. doi:10.1385/1-59259-192-2:115.

37. Leahy SC, Kelly WJ, Li D, Li Y, Altermann E, Lambie SC, et al. The complete genome sequence of Methanobrevibacter sp. AbM4. Stand Genomic Sci. 2013. doi:10.4056/sigs.3977691.

38. Benson DA, Karsch-Mizrachi I, Lipman DJ, Ostell J, Rapp BA, Wheeler DL. GenBank. Nucleic Acids Res. 2000;28:1.

39. Majernik Al, Chong JP. A conserved mechanism for replication origin recognition and binding in archaea. Biochem J. 2008. doi:10.1042/BJ20070213.

40. Takeshima H, Komazaki S, Nishi M, lino M, Kangawa K. Junctophilins: a novel family of junctional membrane complex proteins. Mol Cell. 2000;6:1.

41. Lehnherr H, Maguin E, Jafri S, Yarmolinsky MB. Plasmid addiction genes of bacteriophage P1: doc, which causes cell death on curing of prophage, and phd, which prevents host death when prophage is retained. J Mol Biol. 1993. doi:10.1006/jmbi.1993.1521.

42. Hayes CS, Sauer RT. Toxin-antitoxin pairs in bacteria: killers or stress regulators? Cell. 2003;112:1.

43. Welander PV, Metcalf WW. Mutagenesis of the C1 oxidation pathway in Methanosarcina barkeri: new insights into the Mtr/Mer bypass pathway. J Bacteriol. 2008. doi:10.1128/JB.01424-07.

44. Fricke WF, Seedorf $H$, Henne A, Kruer M, Liesegang $H$, Hedderich $R$, et al. The genome sequence of Methanosphaera stadtmanae reveals why this human intestinal archaeon is restricted to methanol and $\mathrm{H}_{2}$ for methane formation and ATP synthesis. J Bacteriol. 2006. doi:10.1128/ JB.188.2.642-658.2006.

45. Thauer RK, Kaster AK, Seedorf H, Buckel W, Hedderich R. Methanogenic archaea: ecologically relevant differences in energy conservation. Nature Rev Microbiol. 2008. doi:10.1038/nrmicro1931.

46. Baumer S, Ide T, Jacobi C, Johann A, Gottschalk G, Deppenmeier U. The $\mathrm{F}_{420} \mathrm{H}_{2}$ dehydrogenase from Methanosarcina mazei is a redox-driven proton pump closely related to $\mathrm{NADH}$ dehydrogenases. J Biol Chem. 2000. doi:10.1074/jbc.M000650200.

47. Deppenmeier $U$. The membrane-bound electron transport system of Methanosarcina species. J Bioenerg Biomembr. 2004;36:1.

48. Lie TJ, Costa KC, Lupa B, Korpole S, Whitman WB, Leigh JA. Essential anaplerotic role for the energy-converting hydrogenase Eha in hydrogenotrophic methanogenesis. Proc Natl Acad Sci U S A. 2012 doi:10.1073/pnas.1208779109.

49. Welte $\mathrm{C}$, Deppenmeier $\mathrm{U}$. Re-evaluation of the function of the $\mathrm{F}_{420}$ dehydrogenase in electron transport of Methanosarcina mazei. FEBS J. 2011. doi:10.1111/j.1742-4658.2011.08048.x.

50. Deppenmeier U, Johann A, Hartsch T, Merkl R, Schmitz RA, MartinezArias $\mathrm{R}$, et al. The genome of Methanosarcina mazei: evidence for lateral gene transfer between bacteria and archaea. J Mol Microbiol Biotechnol. 2002;4:4.

51. Srinivasan $G$, James CM, Krzycki JA. Pyrrolysine encoded by UAG in Archaea: charging of a UAG-decoding specialized tRNA. Science. 2002. doi:10.1126/science.1069588. 
52. Hao B, Gong W, Ferguson TK, James CM, Krzycki JA, Chan MK. A new UAGencoded residue in the structure of a methanogen methyltransferase. Science. 2002. doi:10.1126/science.1069556.

53. Gaston MA, Zhang L, Green-Church KB, Krzycki JA. The complete biosynthesis of the genetically encoded amino acid pyrrolysine from lysine. Nature. 2011. doi:10.1038/nature09918.

54. Blight SK, Larue RC, Mahapatra A, Longstaff DG, Chang E, Zhao G, et al. Direct charging of tRNA(CUA) with pyrrolysine in vitro and in vivo. Nature. 2004. doi:10.1038/nature02895.

55. Borrel G, Gaci N, Peyret P, O'Toole PW, Gribaldo S, Brugere JF. Unique characteristics of the pyrrolysine system in the 7th order of methanogens: implications for the evolution of a genetic code expansion cassette. Archaea. 2014. doi:10.1155/2014/374146.

56. Seedorf H, Kittelmann S, Janssen PH. Few highly abundant operational taxonomic units dominate within rumen methanogenic archaeal species in New Zealand sheep and cattle. Appl Environ Microbiol. 2015. doi:10.1128/AEM.03018-14.

57. Arbing MA, Chan S, Shin A, Phan T, Ahn CJ, Rohlin L, et al. Structure of the surface layer of the methanogenic archaean Methanosarcina acetivorans. Proc Natl Acad Sci U S A. 2012. doi:10.1073/pnas.1120595109.

58. Borrel G, OToole PW, Harris HM, Peyret P, Brugere JF, Gribaldo S. Phylogenomic data support a seventh order of Methylotrophic methanogens and provide insights into the evolution of Methanogenesis. Genome Biol Evol. 2013. doi:10.1093/gbe/evt128.

59. Dridi B, Fardeau ML, Ollivier B, Raoult D, Drancourt M. Methanomassiliicoccus luminyensis gen. nov., sp. nov., a methanogenic archaeon isolated from human faeces. Int J Syst Evol Microbiol. 2012. doi:10.1099/ijs.0.033712-0.

60. Khan $\mathrm{H}$, Flint SH, Yu PL. Determination of the mode of action of enterolysin A, produced by Enterococcus faecalis B9510. J Appl Microbiol. 2013. doi:10.1111/jam.12240.

61. Kimura M. A simple method for estimating evolutionary rates of base substitutions through comparative studies of nucleotide sequences. J Mol Evol. 1980;16:2.

62. Tamura K, Stecher G, Peterson D, Filipski A, Kumar S. MEGA6: Molecular Evolutionary Genetics Analysis version 6.0. Mol Biol Evol. 2013. doi:10.1093/molbev/mst197.

63. Felsenstein J. Confidence limits on phylogenies: an approach using the Bootstrap. Evolution. 1985. doi:10.2307/2408678.

64. Woese CR, Kandler O, Wheelis ML. Towards a natural system of organisms: proposal for the domains Archaea, Bacteria, and Eucarya. Proc Natl Acad Sci U S A. 1990;87:12.

65. Garrity GM, Holt JG. Phylum All. Euryarchaeota phy. nov. Bergey's Manual ${ }^{\circledR}$ of Systematic Bacteriology. New York: Springer; 2001. p. 211-355.

66. lino T, Tamaki H, Tamazawa S, Ueno Y, Ohkuma M, Suzuki Kl, et al. Candidatus Methanogranum caenicola: a novel methanogen from the anaerobic digested sludge, and proposal of Methanomassiliicoccaceae fam. nov. and Methanomassiliicoccales ord. nov., for a methanogenic lineage of the class Thermoplasmata. Microbes Environ. 2013;28:2

67. Oren A, Garrity GM. List of new names and new combinations previously effectively, but not validly, published. Int J Syst Evol Microbiol. 2013. doi:10.1099/ijs.0.049312-0.

68. Ashburner M, Ball CA, Blake JA, Botstein D, Butler H, Cherry JM, et al. Gene ontology: tool for the unification of biology. The Gene Ontology Consortium. Nature genetics. 2000. doi:10.1038/75556.

69. Altermann E, Klaenhammer TR. GAMOLA: a new local solution for sequence annotation and analyzing draft and finished prokaryotic genomes. OMICS. 2003. doi:10.1089/153623103322246557.

70. Carver T, Thomson N, Bleasby A, Berriman M, Parkhill J. DNAPlotter: circular and linear interactive genome visualization. Bioinformatics (Oxford, England). 2009. doi:10.1093/bioinformatics/btn578.

71. Delcher AL, Salzberg SL, Phillippy AM. Using MUMmer to identify similar regions in large sequence sets. Curr Protoc Bioinformatics. 2003. doi:10.1002/0471250953.bi1003s00

\section{Submit your next manuscript to BioMed Central and we will help you at every step:}

- We accept pre-submission inquiries

- Our selector tool helps you to find the most relevant journal

- We provide round the clock customer support

- Convenient online submission

- Thorough peer review

- Inclusion in PubMed and all major indexing services

- Maximum visibility for your research

Submit your manuscript at www.biomedcentral.com/submit
Biomed Central 